Internat. J. Math. \& Math. Sci.

Vol. 23, No. 1 (2000) 55-63

S0161171200001873

(C) Hindawi Publishing Corp.

\title{
A CURIOUS PROPERTY OF SERIES INVOLVING TERMS OF GENERALIZED SEQUENCES
}

\author{
ODOARDO BRUGIA and PIERO FILIPPONI
}

(Received 13 April 1998 and in revised form 11 September 1998)

\begin{abstract}
Here we are concerned with series involving generalized Fibonacci numbers $U_{n}(p, q)$ and generalized Lucas numbers $V_{n}(p, q)$. The aim of this paper is to find triples $(p, q, r)$ for which the series $U_{n}(p, q) / r^{n}$ and $V_{n}(p, q) / r^{n}$ (for $r$ running from 0 to infinity) are unconcerned at the introduction of the factor $n$. The results established in this paper generalize the known fact that the series $F_{n} / 2^{n}\left(F_{n}\right.$ the $n$th Fibonacci number) and the series $n F_{n} / 2^{n}$ give the same result, namely $-2 / 5$.
\end{abstract}

Keywords and phrases. Generalized Fibonacci numbers, power series.

2000 Mathematics Subject Classification. 30B10, 11B39, 26 D05.

1. Introduction. First, we define the sequences under study, then we explain the aim of our paper.

1.1. The generalized sequences. For $a, b, p, q$ arbitrary real numbers (in particular, integers), in the notation of Horadam [3] write

$$
W_{n}:=W_{n}(a, b ; p, q),
$$

meaning that

$$
W_{0}=a, \quad W_{1}=b, \quad W_{n}=p W_{n-1}-q W_{n-2} \quad \text { for } n \geq 2 .
$$

In particular, here we are concerned with the numbers

$$
U_{n}(p, q)=U_{n}:=W_{n}(0,1 ; p, q) \quad \text { and } \quad V_{n}(p, q)=V_{n}:=W_{n}(2, p ; p, q) .
$$

Observe that $U_{n}(1,-1)=F_{n}$ and $V_{n}(1,-1)=L_{n}$ are the $n$th Fibonacci and Lucas number, respectively. The Binet forms for $U_{n}$ and $V_{n}$ are

$$
U_{n}=\frac{\left(\alpha^{n}-\beta^{n}\right)}{\sqrt{\Delta}} \quad \text { and } \quad V_{n}=\alpha^{n}+\beta^{n},
$$

where

$$
\begin{gathered}
\Delta=p^{2}-4 q, \\
\alpha=\frac{(p+\sqrt{\Delta})}{2} \quad \text { and } \quad \beta=\frac{(p-\sqrt{\Delta})}{2}
\end{gathered}
$$

are the roots, assumed distinct, of the equation $x^{2}-p x+q=0$. Observe that (1.6) yields the two relations

$$
\alpha+\beta=p \quad \text { and } \quad \alpha \beta=q
$$


which will be widely used throughout this paper. As usual, we require that

$$
\Delta>0
$$

so that $\alpha, \beta$, and $\sqrt{\Delta}$ are real, where $\alpha \neq \beta$ as assumed. We assume also that

$$
p q \neq 0 \text {. }
$$

1.2. Motivation and aim of the paper. We were amazed at the equality

$$
\sum_{n=0}^{\infty} \frac{F_{n}}{(-2)^{n}}=\sum_{n=0}^{\infty} \frac{n F_{n}}{(-2)^{n}} \quad\left(=-\frac{2}{5}\right)
$$

which is reported in [2, (4.20)] as a by-product result. One can immediately observe how the series on the left-hand side of (1.10) is, quite surprisingly, unconcerned at the introduction of the factor $n$. Our mathematical curiosity led us to seek analogs of (1.10) that involve the more general sequences $U_{n}$ and $V_{n}$ defined by (1.3). In fact, the aim of this paper is to find triples $(p, q, r)$ of real numbers (with $r \neq 0$ ) for which the equality

$$
\sum_{n=0}^{\infty} \frac{W_{n}(p, q)}{r^{n}}=\sum_{n=0}^{\infty} \frac{n W_{n}(p, q)}{r^{n}}
$$

(here $W$ stands for either $U$ or $V$ ) holds true. The cases where $p$ and $q$ are integers and $r$ is rational (possibly an integer) are treated as particular instances. While the case $W \equiv U$ (Section 3 ) is readily solved, some particular aspects of the case $W \equiv V$ (Section 4) are worth investigating (Subsections 4.1 and 4.2). Several numerical examples illustrate the theoretical results whose proofs are given in full, except for the detailed discussions on certain inequalities which are omitted for the sake of brevity.

2. Preliminary results. The following two lemmas will be needed in Sections 3 and 4 , respectively.

LEMMA 2.1. If $x$ and $y$ are arbitrary complex numbers such that $x \neq y$,

$$
|x|<1 \quad \text { and } \quad|y|<1 \text {, }
$$

then the equality

$$
\sum_{n=0}^{\infty} x^{n}-\sum_{n=0}^{\infty} y^{n}=\sum_{n=0}^{\infty} n x^{n}-\sum_{n=0}^{\infty} n y^{n}
$$

is satisfied if and only if

$$
x+y=2 x y .
$$

To prove Lemma 2.1, we show that (2.3) is nothing but an equivalent form of (2.2). The same technique will be used in the proofs of Lemma 2.2 and Theorems 3.1 and 4.1.

Proof of Lemma 2.1. Using the geometric series formula, (2.2) yields

$$
\frac{1}{1-x}-\frac{1}{1-y}=\frac{x}{(1-x)^{2}}-\frac{y}{(1-y)^{2}}
$$


which, in turn, can be equivalently rewritten as

$$
\begin{aligned}
& -\frac{1-x-1}{(1-x)^{2}}-\frac{1}{1-x}+\frac{1-y-1}{(1-y)^{2}}+\frac{1}{1-y}=0, \\
& \left(\frac{1}{1-x}\right)^{2}-\frac{2}{1-x}+\frac{2}{1-y}-\left(\frac{1}{1-y}\right)^{2}=0, \\
& \left(\frac{1}{1-x}-\frac{1}{1-y}\right)\left(\frac{1}{1-x}+\frac{1}{1-y}-2\right)=0 .
\end{aligned}
$$

Since the first factor on the left-hand side of (2.5) cannot vanish as $x \neq y$ by hypothesis, let us equate to zero the second factor thus getting the following equation:

$$
1-y+1-x-2(1-x)(1-y)=0
$$

which yields the desired result (2.3).

LEMMA 2.2. If $x$ and $y$ are as in the statement of Lemma 2.1 ( $x=y$ allowed), then the equality

$$
\sum_{n=0}^{\infty} x^{n}+\sum_{n=0}^{\infty} y^{n}=\sum_{n=0}^{\infty} n x^{n}+\sum_{n=0}^{\infty} n y^{n}
$$

is satisfied if and only if

$$
\left(\frac{x}{1-x}\right)^{2}+\left(\frac{y}{1-y}\right)^{2}=2 .
$$

Proof. Similar to the proof of Lemma 2.1, (2.7) yields

$$
-\frac{1-x-1}{(1-x)^{2}}-\frac{1}{1-x}-\frac{1-y-1}{(1-y)^{2}}-\frac{1}{1-y}=0
$$

which, in turn, can be equivalently rewritten as

$$
\begin{gathered}
\left(\frac{1}{1-x}\right)^{2}-\frac{2}{1-x}+1+\left(\frac{1}{1-y}\right)^{2}-\frac{2}{1-y}+1=2, \\
\left(\frac{1}{1-x}-1\right)^{2}+\left(\frac{1}{1-y}-1\right)^{2}=2,
\end{gathered}
$$

whence (2.8) is immediately obtained.

REMARK 2.1. In the special case $x=y$, (2.7) reduces to $\sum_{0}^{\infty} x^{n}=\sum_{0}^{\infty} n x^{n}$ whose solution is $x=1 / 2$.

\section{Series involving the numbers $U_{n}$}

THEOREM 3.1. If

with

$$
r=\frac{2 q}{p}
$$

$$
q<-\frac{3 p^{2}}{4},
$$


then

$$
\sum_{n=0}^{\infty} \frac{U_{n}(p, q)}{r^{n}}=\sum_{n=0}^{\infty} \frac{n U_{n}(p, q)}{r^{n}} \text { for all nonzero } p
$$

REMARK 3.1. Using (1.4), (1.5), (1.6), (3.1), and the geometric series formula, it is readily seen that the sums of the series in (3.3) equal $2 p /\left(4 q-p^{2}\right)=-2 p / \Delta$.

Proof of Theorem 3.1. Use the Binet form (1.4) to rewrite (3.3) as

$$
\sum_{0}^{\infty}\left(\frac{\alpha}{r}\right)^{n}-\sum_{0}^{\infty}\left(\frac{\beta}{r}\right)^{n}=\sum_{0}^{\infty} n\left(\frac{\alpha}{r}\right)^{n}-\sum_{0}^{\infty} n\left(\frac{\beta}{r}\right)^{n} .
$$

From Lemma 2.1 we know that (3.4) holds true if $(\alpha / r)+(\beta / r)=2 \alpha \beta / r^{2}$, that is, if

$$
\frac{p}{r}=\frac{2 q}{r^{2}} \quad \text { from (1.7), }
$$

whence condition (3.1) appears to be sufficient.

Condition (1.8) is clearly satisfied if $q / p^{2}<1 / 4$, that is, if

$$
q<\frac{p^{2}}{4} .
$$

Further, after replacing $x$ (respectively $y$ ) by $\alpha / r$ (respectively $\beta / r$ ) in conditions (2.1), and taking the value of $r$ given by (3.1) into account, it becomes clear that we must have

$$
-1<\frac{1 \pm \sqrt{1-\left(4 q / p^{2}\right)}}{4 q / p^{2}}<1 .
$$

It is not hard to prove that the necessary and sufficient condition for inequalities (3.7) to be satisfied is that $q / p^{2}<-3 / 4$. In other words, we must have

$$
q<-\frac{3 p^{2}}{4} .
$$

Combining (3.6) and (3.8) yields condition (3.2). This completes the proof.

3.1. Numerical examples. Letting $p=6$ and $q=-28$ in (3.1) (thus getting $r=$ $-28 / 3$ ) yields

$$
\sum_{0}^{\infty} \frac{U_{n}(6,-28)}{(-28 / 3)^{n}}=\sum_{0}^{\infty} \frac{n U_{n}(6,-28)}{(-28 / 3)^{n}}=-\frac{3}{37},
$$

whereas letting $p=5$ and $q=-20(r=-8)$ yields

$$
\sum_{0}^{\infty} \frac{U_{n}(5,-20)}{(-8)^{n}}=\sum_{0}^{\infty} \frac{n U_{n}(5,-20)}{(-8)^{n}}=-\frac{2}{21} .
$$

The analog of (1.10) for the numbers $G_{n}(m)$ defined in [1] as $G_{n}(m):=U_{n}(1,-m)$, $m=1,2,3, \ldots$, is

$$
\sum_{0}^{\infty} \frac{G_{n}(m)}{(-2 m)^{n}}=\sum_{0}^{\infty} \frac{n G_{n}(m)}{(-2 m)^{n}}=-\frac{2}{4 m+1} .
$$


Observe that (3.11) and (1.10) coincide for $m=1$ whereas, for $m=2$ (see [4]), (3.11) is the Jacobsthal-analog of (1.10).

It is worth noting that the analog of (1.10) cannot be found for Pell numbers [5] $P_{n}:=U_{n}(2,-1)$. In fact, since $-1>-3 \cdot 2^{2} / 4$, condition (3.2) is not satisfied whereas in all the previous examples it is.

\section{Series involving the numbers $V_{n}$}

THEOREM 4.1. If $s$ is a real number subject to

$$
\begin{gathered}
s<\frac{-(2+\sqrt{7})}{2} \quad \text { or } \quad s>1, \\
r=s p, \\
q=\frac{s p^{2}}{2}\left(\frac{s^{2}}{3 s-1}-s+1\right),
\end{gathered}
$$

then

$$
\sum_{n=0}^{\infty} \frac{V_{n}(p, q)}{r^{n}}=\sum_{n=0}^{\infty} \frac{n V_{n}(p, q)}{r^{n}} \text { for all nonzero } p
$$

REMARK 4.1. From (4.1) and (4.3), we can see that $q$ is negative, except when $1<$ $s<1+\sqrt{2} / 2$. Using (1.4), (1.5), (1.6), (4.2), (4.3), and the geometric series formula, it is readily seen that the sums of the series in (4.4) equal $2(3 s-1) /(2 s-1)$.

Proof OF THEOREM 4.1. Use the Binet form (1.4) to rewrite (4.4) as

$$
\sum_{0}^{\infty}\left(\frac{\alpha}{r}\right)^{n}+\sum_{0}^{\infty}\left(\frac{\beta}{r}\right)^{n}=\sum_{0}^{\infty} n\left(\frac{\alpha}{r}\right)^{n}+\sum_{0}^{\infty} n\left(\frac{\beta}{r}\right)^{n} .
$$

From Lemma 2.2, we know that (4.5) holds true if

$$
\left[\frac{\alpha}{r-\alpha}\right]^{2}+\left[\frac{\beta}{r-\beta}\right]^{2}=2
$$

By using (1.7) along with the identity $\alpha^{2}+\beta^{2}=p^{2}-2 q$, after some simple manipulations (4.6) can be rewritten as

$$
-p^{2} r-2 r^{3}+4 p r^{2}-6 q r+2 p q=0
$$

whence

$$
q=\frac{-r\left(2 r^{2}-4 p r+p^{2}\right)}{2(3 r-p)}
$$

Now impose condition (4.2) (namely, $r=s p$ with $s$ subject to (4.1)) in (4.8) to get

$$
q=\frac{p^{2} s\left(4 s-2 s^{2}-1\right)}{2(3 s-1)}=\frac{s p^{2}}{2}\left(\frac{s^{2}}{3 s-1}-s+1\right),
$$

as expected.

From (4.9) above, the quantity $\Delta$ defined by (1.5) can be expressed as

$$
\Delta=\frac{p^{2}\left(4 s^{3}-8 s^{2}+5 s-1\right)}{3 s-1}=\frac{p^{2}(s-1)(2 s-1)^{2}}{3 s-1},
$$


so that condition (1.8) (namely, $\Delta>0$ ) is satisfied if either

$$
s<\frac{1}{3} \quad \text { or } \quad s>1 .
$$

Further, after replacing $x$ (respectively $y$ ) by $\alpha / \gamma$ (respectively $\beta / r$ ) in conditions (2.1), and taking the value of $r$ and $\Delta$ (given by (4.2) and (4.10), respectively) into account, it becomes clear that we must have

$$
-1<\frac{1 \pm(2 s-1) \sqrt{(s-1) /(3 s-1)}}{2 s}<1 .
$$

After some tedious calculations, one sees that the inequalities (4.12) above are satisfied if either

$$
s<\frac{-(2+\sqrt{7})}{2} \quad \text { or } \quad s>\frac{1}{2} .
$$

Combining (4.11) and (4.13) yields (4.1). This completes the proof.

4.1. The integrality of $q$, and numerical examples. If the real number $s$ (see (4.1)) is a rational number $s=m / d$ with $\operatorname{gcd}(m, d)=1(s$ an integer if $d=1)$, then (4.3) becomes

$$
q=\frac{m p^{2}}{2 d}\left(-\frac{m}{d} \cdot \frac{2 m-d}{3 m-d}+1\right)
$$

By (4.3), for a given integer $p$ it is clear that $q$ is not necessarily an integer.

As a numerical example, if we let $s=3 / 2$ (see the first part of Remark 4.1) and $p=14$, then from (4.2) and (4.14) we get $r=q=21$, so that

$$
\sum_{0}^{\infty} \frac{V_{n}(14,21)}{21^{n}}=\sum_{0}^{\infty} \frac{n V_{n}(14,21)}{21^{n}}=\frac{7}{2} .
$$

Let us conclude this subsection by finding the analog of (1.10) for the Lucas numbers. If we put the Lucas parameters $p=1$ and $q=-1$ in (4.3), then we get the cubic equation

$$
s^{3}-2 s^{2}-\frac{5}{2} s+1=0 .
$$

The values of $r(=p s=1 \cdot s=s)$ for which (4.4) holds are clearly given by all the (real) solutions of (4.16) that fulfill (4.1). It can be seen, with the aid of a computer, that only the root

$$
\rho=2.771784 \ldots
$$

has this property. Hence, we get

$$
\sum_{0}^{\infty} \frac{L_{n}}{\rho^{n}}=\sum_{0}^{\infty} \frac{n L_{n}}{\rho^{n}}=3.220091 \ldots .
$$

4.2. A question and its answer. By observing (4.4), a question arises quite naturally.

QUESTION. For given $p$ and $q$, how many distinct values of $r$ do there exist for which (4.4) holds?

The answer is given in the following proposition. 
Proposition. For given $p$ and $q$, there exist at most two distinct values of $r$ for which (4.4) holds.

Proof. By putting $q / p^{2}=a$ in (4.3), one gets the cubic equation in the unknown $s$

$$
s^{3}-2 s^{2}+\left(3 a+\frac{1}{2}\right) s-a=0 .
$$

By imposing that $s_{0}$ is a real root of (4.19), one obtains

$$
a=\frac{s_{0}\left(-2 s_{0}^{2}+4 s_{0}-1\right)}{2\left(3 s_{0}-1\right)},
$$

whence, by substituting (4.20) in (4.19), one can write

$$
\left(s-s_{0}\right)\left[s^{2}+\left(s_{0}-2\right) s-\frac{2 s_{0}^{2}-4 s_{0}+1}{2\left(3 s_{0}-1\right)}\right]=0,
$$

after some simple manipulations. The discriminant $\delta$ of the second-degree factor above, given by

$$
\delta=\frac{3 s_{0}^{3}-9 s_{0}^{2}+8 s_{0}-2}{3 s_{0}-1}=\frac{3\left(s_{0}-1\right)\left[s_{0}-(3-\sqrt{3}) / 3\right]\left[s_{0}-(3+\sqrt{3}) / 3\right]}{3 s_{0}-1},
$$

is nonnegative if either

$$
s_{0}<\frac{1}{3}, \quad \frac{3-\sqrt{3}}{3} \leq s_{0} \leq 1, \quad \text { or } \quad s_{0} \geq \frac{3+\sqrt{3}}{3} .
$$

Hence, the further real roots $s_{1}$ and $s_{2}$ of (4.19) are

$$
s_{1}=\frac{\left(2-s_{0}+\sqrt{\delta}\right)}{2} \quad \text { and } \quad s_{2}=\frac{\left(2-s_{0}-\sqrt{\delta}\right)}{2}
$$

from (4.21), (4.22), and (4.23).

We prove that, if $s_{0}$ is chosen in such a way that

$$
s_{0}<\frac{-2+\sqrt{7}}{2} \quad \text { or } \quad s_{0}>\frac{3+\sqrt{3}}{3}
$$

obtained by combining (4.1) and (4.23), then at most one of the roots $s_{1}$ and $s_{2}$ given by (4.24) satisfies (4.1). After a good deal of algebraic manipulation, one obtains the following:

(a) $s_{1}$ and $s_{2}$ cannot be both greater than 1 because $s_{1}>1 \rightarrow s_{0}<1 / 3$ and $s_{2}>1 \rightarrow$ $s_{0}>1 / 3$. Let us confine ourselves to sketch the proof only for the first implication. The second one can be proved in a similar way. From (4.24) we get the implication $s_{1}>1 \rightarrow s_{0}<\sqrt{\delta}$ whose right-hand side is satisfied if $s_{0}<0$. If $s_{0}>0$, from (4.22) we can rewrite the implication above as $s_{1}>1 \rightarrow\left(2 s_{0}-1\right)^{2} /\left(3 s_{0}-1\right)<0$ whence $s_{1}>1 \rightarrow 3 s_{0}-1<0$, that is, $s_{1}>1 \rightarrow s_{0}<1 / 3$.

(b) $s_{1}$ and $s_{2}$ cannot be both less than $-(2+\sqrt{7}) / 2$ because $s_{1}$ cannot be.

(c) After observing that $s_{1}>s_{2}$ (see (4.24)), it is patent that the remaining possibility for both $s_{1}$ and $s_{2}$ to satisfy (4.1) is

(i) $s_{1}>1$,

(ii) $s_{2}<-(2+\sqrt{7}) / 2$. 
Condition (i) is satisfied for $s_{0}<1 / 3$ (see (a)) whereas condition (ii) is satisfied for either $s_{0}>t_{1}$ or $t_{2}<s_{0}<1 / 3$, where

$$
t_{1}=3.991448 \ldots \text { and } \quad t_{2}=0.331427 \ldots
$$

are the roots of the equation $(16+6 \sqrt{7}) s_{0}^{2}-(69+26 \sqrt{7}) s_{0}+21+8 \sqrt{7}=0$ obtained by imposing $s_{2}=-(2+\sqrt{7}) / 2$.

Now, from (c) it is evident that the inequalities $s_{1}>1$ and $s_{2}<-(2+\sqrt{7}) / 2$ are simultaneously satisfied if $t_{2}<s_{0}<1 / 3$, this contradicts (4.25)! Taking (c) and (4.25) into account, it follows that (4.4) holds either for

$$
r=r_{0}=p s_{0} \quad \text { and } \quad r=r_{1}=p s_{1} \quad\left(\text { when } s_{0}<\frac{-(2+\sqrt{7})}{2}\right),
$$

or for

$$
r=r_{0}=p s_{0} \quad\left(\text { when } \frac{3+\sqrt{3}}{3}<s_{0}<t_{1}\right)
$$

or for

$$
r=r_{0}=p s_{0} \quad \text { and } \quad r=r_{2}=p s_{2} \quad\left(\text { when } s_{0}>t_{1}\right)
$$

As a numerical example, let $s_{0}=10\left(>t_{1}\right)$ in (4.22) thus getting $s_{1,2}=(-8 \pm$ $33 \sqrt{2 / 29}) / 2$, and observe that $s_{2}$ satisfies (4.1) whereas $s_{1}$ does not. Further, let $p=29$ and $s=s_{0}$ in (4.3) thus getting $q=-23345$, and $r_{0}=p s_{0}=290$ and $r_{2}=p s_{2}=29 s_{2}=$ $(-232-957 \sqrt{2 / 29}) / 2$. Put the above values of $p, q, r_{0}$ and $r_{2}$ in (4.4), and write

$$
\begin{aligned}
& \sum_{0}^{\infty} \frac{V_{n}(29,-23345)}{290^{n}}=\sum_{0}^{\infty} \frac{n V_{n}(29,-23345)}{290^{n}}=\frac{58}{19}, \\
& \sum_{0}^{\infty} \frac{V_{n}(29,-23345)}{r_{2}^{n}}=\sum_{0}^{\infty} \frac{n V_{n}(29,-23345)}{r_{2}^{n}} \approx 2.9433 .
\end{aligned}
$$

Finally, we observe that, if we replace $V_{n}(p, q)$ by $H_{n}(m):=V_{n}(1,-m), m=1,2,3, \ldots$, (see [1]) in (4.4), then this equality holds only for

$$
r=p s_{0}=s_{0}=\frac{2[\sqrt{(18 m+5) / 2} \cos (\gamma / 3)+1]}{3}
$$

with

$$
\gamma=\arccos \left[\frac{(27 m+7) \sqrt{2 /(18 m+5)}}{(18 m+5)}\right],
$$

if $1 \leq m \leq 3$ (cf. the Lucas case (4.17) and (4.18) for $m=1$ ), whereas the above said equality holds for both $r=s_{0}$ and $r=p s_{2}=s_{2}$ (see (4.24)) if $m \geq 4$.

5. A concluding remark. It is worth noting that the results established in Section 2 allow us to obtain some interesting identities involving circular and hyperbolic functions. For example, we invite the interested reader to use Lemma 2.1 along with the 
exponential representation of $\sin \omega$ to prove that, if $|r|>1$ and $\omega=\arccos (1 / r)$, then

$$
\sum_{n=0}^{\infty} \frac{\sin (n \omega)}{r^{n}}=\sum_{n=0}^{\infty} \frac{n \sin (n \omega)}{r^{n}}=\frac{r \sin \omega}{r^{2}-2 r \cos \omega+1} .
$$

As a numerical example, letting $r=2$ in (5.1) yields

$$
\sum_{0}^{\infty} \frac{\sin (n \pi / 3)}{2^{n}}=\sum_{0}^{\infty} \frac{n \sin (n \pi / 3)}{2^{n}}=\frac{\sqrt{3}}{3} .
$$

The cosine-analog of (5.1) involves the use of Lemma 2.2.

ACKNOWLEDGEMENT. This work has been carried out in the framework of an agreement between the Italian PT Administration (Istituto Superiore PT) and the Fondazione Ugo Bordoni. The authors wish to thank the anonymous referees whose suggestions led to a substantial improvement of the presentation of the paper.

\section{REFERENCES}

[1] P. Filipponi, A note on a class of Lucas sequences, Fibonacci Quart. 29 (1991), no. 3, 256263. MR 92d:11009. Zbl 734.11017.

[2] _ Some binomial Fibonacci identities, Fibonacci Quart. 33 (1995), no. 3, 251-257. MR 96c:11020. Zbl 843.11010.

[3] A. F. Horadam, Basic properties of a certain generalized sequence of numbers, Fibonacci Quart. 3 (1965), 161-176. MR 32\#4074. Zbl 131.04103.

[4] _ Jacobsthal representation numbers, Fibonacci Quart. 34 (1996), no. 1, 40-54. MR 96j:11027. Zbl 869.11013.

[5] A. F. Horadam and Bro. J. M. Mahon, Pell and Pell-Lucas polynomials, Fibonacci Quart. 23 (1985), no. 1, 7-20. MR 86h:11017. Zbl 557.10011.

BRugia: Fondazione Ugo Bordoni, Via B. CASTiglione 59, I-00142 Roma, Italy

FilipPoni: FondAZIONE Ugo BoRdoni, Via B. CASTiglione 59, I-00142 Roma, ITALY

E-mail address: filippo@fub.it 


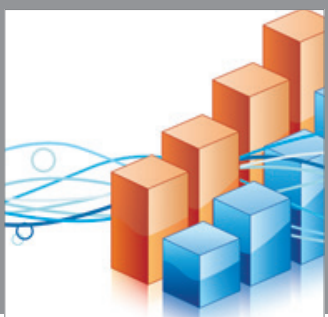

Advances in

Operations Research

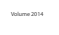

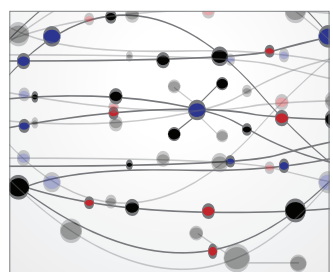

\section{The Scientific} World Journal
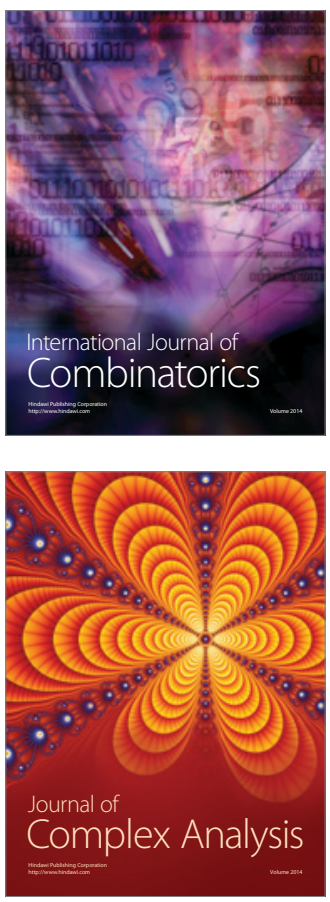

International Journal of

Mathematics and

Mathematical

Sciences
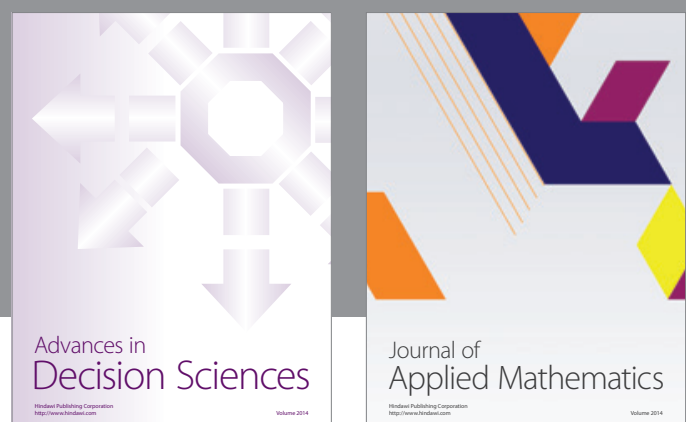

Journal of

Applied Mathematics
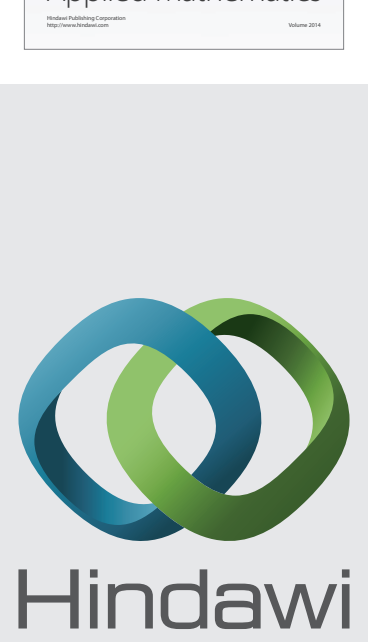

Submit your manuscripts at http://www.hindawi.com
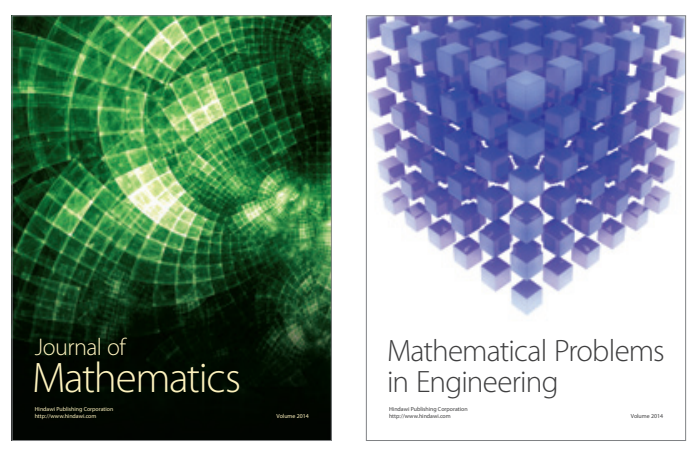

Mathematical Problems in Engineering
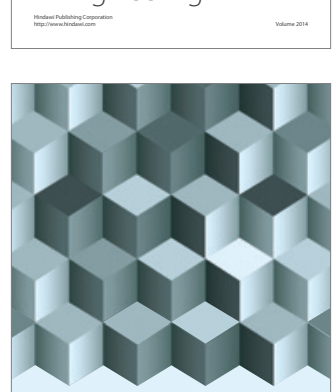

Journal of

Function Spaces
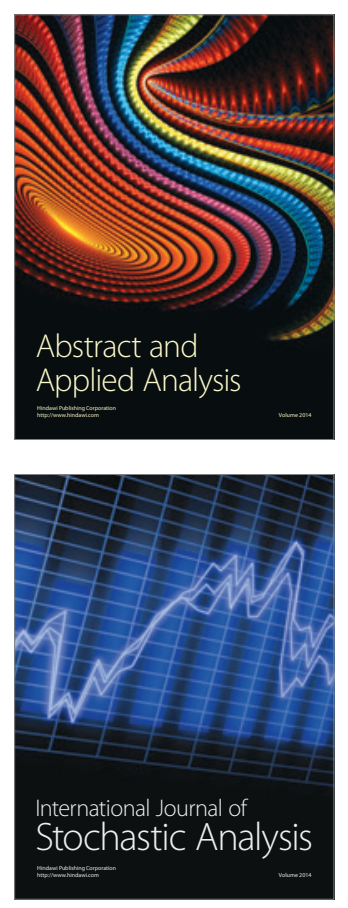

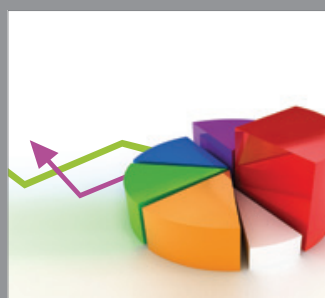

ournal of

Probability and Statistics

Promensencen
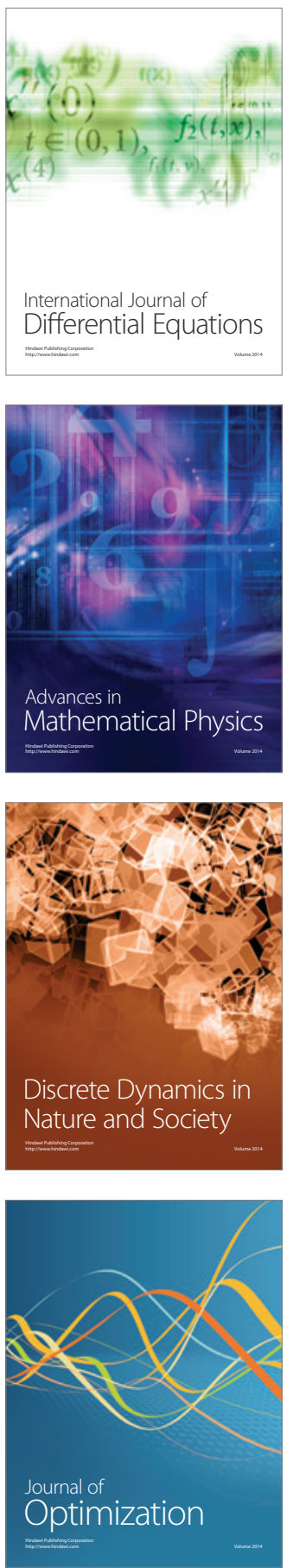